\title{
Properties of Dense Strange Hadronic Matter with Quark Degrees of Freedom
}

\author{
I. Zakout ${ }^{1,2}$, H. R. Jaqaman ${ }^{1}$, H. Stöcker ${ }^{2}$ and W. Greiner ${ }^{2}$ \\ ${ }^{1}$ Department of Physics, Bethlehem University, P.O. Box 9, Bethlehem, Palestinian Authority \\ ${ }^{2}$ Institut für Theoretische Physik, J. W. Goethe Universität, Frankfurt am Main, Germany
}

(May 1, 2001)

\begin{abstract}
The properties of strange hadronic matter are studied in the context of the modified quark-meson coupling model using two substantially different sets of hyperon-hyperon $(Y Y)$ interactions. The first set is based on the Nijmegen hard core potential model $\mathrm{D}$ with slightly attractive $Y Y$ interactions. The second potential set is based on the recent $S U(3)$ extension of the Nijmegen soft-core potential NSC97 with strongly attractive $Y Y$ interactions which may allow for deeply bound hypernuclear matter. The results show that, for the first potential set, the $\Sigma$ hyperon does not appear at all in the bulk at any baryon density and for all strangeness fractions. The binding energy curves of the resulting $N \Lambda \Xi$ system vary smoothly with density and the system is stable (or metastable if we include the weak force). However, the situation is drastically changed when using the second set where the $\Sigma$ hyperons appear in the system at large baryon densities above a critical strangeness fraction. We find strange hadronic matter undergoes a first order phase transition from a $N \Lambda \Xi$ system to a $N \Sigma \Xi$ for strangeness fractions $f_{S}>1.2$ and baryonic densities exceeding twice ordinary nuclear matter density. Furthermore, it is found that the system built of $N \Sigma \Xi$ is deeply bound. This phase transition affects significantly the equation of state which becomes much softer and a substantial drop in energy density and pressure are detected as the phase transition takes place.
\end{abstract}

PACS:21.65.+f, 24.85.+p, 12.39Ba 


\section{INTRODUCTION}

Extremely dense strange quark matter with a strangeness fraction of order one and charge neutrality has been suggested to be the absolutely stable form of matter at high densities (or at least to be metastable because of the weak interaction) [1] 네. Finite chunks of strange quark matter with large strangeness fractions, the so-called strangelets, are predicted to be more stable than normal nuclei [3,507]. Recent theoretical and experimental searches for strange quark matter can be found in Refs. 8,96

On the other hand, metastable strange systems with strangeness fractions of order one and charge neutrality might also exist in the hadronic phase at moderate values of density, between two and three times nuclear matter density. The properties of metastable exotic multihypernuclear objects with $\Lambda$ and $\Xi$ hyperons reveal quite similar features as the strangelets proposed as a unique signature for quark-gluon plasma formation in heavy ion collisions [10]. The equilibrium between the quark and hadronic phases has been studied in [11]. Strange hadronic matter in bulk has been discussed by Glendenning [12].

It is found that in an extended mean field theory, a large class of bound multistrange objects formed from combinations of $(N, \Lambda, \Xi)$ baryons are stable against strong decay. The presence of filled $\Lambda$ orbitals blocks the strong decay $\Xi N \rightarrow \Lambda \Lambda$ [13]. The maximal binding energy per baryon of $E_{B} / A \approx-21 \mathrm{MeV}$ occurs at a strangeness fraction or strangeness per baryon $f_{s} \approx 1.0-1.2$, charge per baryon $f_{q} \approx-0.1-0.0$ and baryon density 2.5-3 times that of ordinary nuclei [14]. It is comparable to that of hypothetically stable strange quark matter ("strangelets"), which has a binding energy per particle $E_{B} / A \approx-10$ to $-20 \mathrm{MeV}$.

The predicted phenomenon of metastability of strange hadronic matter and the actual values of the binding energy depend specifically on the partly unknown hyperon potentials assumed in dense matter. Some studies used basically Brueckner-Hartree-Fock (BHF) calculations with different Nijmegen soft core potentials [15, 16]. Other studies [14,17,.18] extend relativistic mean field theory (RMF) from ordinary nuclei $\left(f_{S}=0\right)$ to multistrange nuclei with $\left(f_{S} \neq 0\right)$ with the attractive $Y Y$ interaction of the Nijmegen potential Model D [19].

Recently, Stoks and Lee [20] have studied strange hadronic matter using BHF theory and $G$ matrices for coupled baryon channels using an $S U(3)$ extension [21]23] of the Nijmegen soft core NSC97 potential from the $S=0,-1$ sector into the unexplored $S=-2,-3,-4$ sector. They have shown that $N \Lambda \Xi$ systems are only loosely bound and that charge neutral strangeness-rich hadronic systems are unlikely to exist in nature. Unfortunately their procedure is not self-consistent due to the constraint of equal hyperon fractions that they impose and hence does not give the minimum energy [24].

Schaffner-Bielich and Gal [24] have carried out unconstrained RMF calculations and have found larger binding energies. For small strangeness fractions $f_{S} \leq 1$, strange hadronic matter is mainly composed of $N \Lambda \Xi$ and the calculated binding energy closely follows that calculated by using the hyperon potentials of the earlier versions of the soft core Nijmegen potential. For larger strangeness fractions $f_{S} \geq 1$, the calculated binding energy increases substantially due to a phase transition into $N \Sigma \Xi$ dominated matter.

In this work we use the quark-meson coupling Model (QMC) to study strange hadronic matter. The QMC model uses the quark degrees of freedom explicitly by coupling the scalar $\sigma$ and vector $\omega$ mesonic fields directly to the up and down quarks inside the hadrons which are treated as non-overlapping MIT bags. It was first proposed by Guichon [2] and has been 
used in various nuclear calculations [26,27]. More recently it has been modified to include a medium-dependent bag parameter coupled to the scalar $\sigma$ field [28,29]. This coupling is motivated by invoking the nontopological soliton model for the nucleon [30]. Quark deconfinement in hot nuclear matter and the phase transition from the hadronic phase to the quark-gluon plasma have been studied in this model [31,32]. This modified quark-meson coupling model (MQMC) 28,29 has been extended to the study of strange hadronic matter by introducing additional scalar $\sigma^{*}(s \bar{s})$ and vector $\phi(s \bar{s})$ mesonic fields which couple only to the strange quark. This extended model has been used to study the properties of hot hypernuclear matter [33] and neutron star matter [34].

We assume strange hadronic matter to consist of the baryon members of the SU(3) octet and decuplet. The octet is comprised of the nucleons and the $\Lambda, \Sigma$ and $\Xi$ hyperons while the decuplet contains the $\Delta, \Sigma, \Xi$ and $\Omega$ baryons. The decuplet baryons are however not found to have a significant contribution in the current calculations at zero temperature and are henceforth dropped from our formalism. We studied this system with two sets of hyperon-hyperon $(Y Y)$ potentials, the first set is determined from the Nijmegen hard-core potential Model D and the second set corresponds to the potentials obtained in a recent SU(3) extension of the Nijmegen soft-core potential Model NSC97. The differences between these sets are essentially attributable to the extremely attractive $\Sigma \Sigma$ and $\Xi \Xi$ interactions in the second set which allow the possibility of deeply bound nuclear matter with hyperons. We also consider these two potential models in the context of the QMC model with medium independent bag constant.

The current paper is organized as follows. In section II we describe the details of the MQMC model, in section III we show how we fit the various parameters in the model and in section IV we present and discuss our results. Finally we summarize our conclusions in section $\mathrm{V}$.

\section{THE MQMC MODEL}

In the QMC model, the quark field $\psi_{q}(\vec{r}, t)$ inside a bag of radius $R_{i}$ representing a baryon of species $i$ satisfies the Dirac equation

$$
\left[i \gamma^{\mu} \partial_{\mu}-m_{q}^{0}+\left(g_{q \sigma} \sigma-g_{q \omega} \omega_{\mu} \gamma^{\mu}\right)+\left(g_{q \sigma^{*}} \sigma^{*}-g_{q \phi} \phi_{\mu} \gamma^{\mu}\right)\right] \psi_{q}(\vec{r}, t)=0
$$

where the quark is assumed to couple directly to the scalar and vector meson fields and $m_{q}^{0}$ is the current mass of a quark of flavor $q$. The current quark masses are taken, for the up and down flavor quarks, to be $m_{u}=m_{d}=0$ while for the strange flavor $m_{s}=150 \mathrm{MeV}$ Inclusion of small current quark masses for the nonstrange flavors or other values for the strange flavor leads only to small numerical refinements of the present results. In the mean field approximation the meson fields are treated classically and the space like components of the vector fields vanish for infinite systems due to rotational invariance. As a result $\omega_{\mu} \gamma^{\mu}=<\omega_{0}>\gamma^{0}=\omega \gamma^{0}$ and $\phi_{\mu} \gamma^{\mu}=<\phi_{0}>\gamma^{0}=\phi \gamma^{0}$. The nonstrange (up and down) flavor quarks are coupled to the scalar $\sigma(550)$ and vector $\omega(780)$ mesons while the strange flavor quarks are coupled to $\sigma^{*}(975)$ and $\phi(1020)$.

For a given value of the bag radius $R_{i}$ for baryon species $i$ and the scalar fields $\sigma$ and $\sigma^{*}$,

the quark momentum $x_{q}^{i}$ is determined by the boundary condition of confinement which, for quarks of flavor $q$ in a spherical bag, reduces to $j_{0}\left(x_{q}^{i}\right)=\beta_{q} j_{1}\left(x_{q}^{i}\right)$, where 


$$
\beta_{q}=\sqrt{\frac{\Omega_{q}^{i}\left(\sigma, \sigma^{*}\right)-R_{i} m_{q}^{*}}{\Omega_{q}^{i}\left(\sigma, \sigma^{*}\right)+R_{i} m_{q}^{*}}}
$$

We have defined the effective quark mass inside the bag as

$$
m_{q}^{*}=m_{q}^{0}-g_{q \sigma} \sigma-g_{q \sigma^{*}} \sigma^{*}
$$

and the effective quark energy is given by

$$
\Omega_{q}^{i}\left(\sigma, \sigma^{*}\right) / R_{i}=\sqrt{\left(x_{q} / R_{i}\right)^{2}+m_{q}^{* 2}} .
$$

The bag energy for baryon species $i$ is given by

$$
E_{b a g}^{i}=\sum_{q}^{3} n_{q} \frac{\Omega_{q}^{i}\left(\sigma, \sigma^{*}\right)}{R_{i}}-\frac{Z_{i}}{R_{i}}+\frac{4 \pi}{3} R_{i}^{3} B_{i}\left(\sigma, \sigma^{*}\right),
$$

where $\frac{Z_{i}}{R_{i}}$ term is the zero-point energy of the quarks and $B_{i}\left(\sigma, \sigma^{*}\right)$ is the medium-dependent bag parameter. In the simple QMC model, the bag parameter is taken as $B_{0}$ corresponding to its value for a free baryon. The medium effects are taken into account in the MQMC model [28,29] by coupling the bag parameter to the scalar meson fields. In the present work we use the following generalized ansatz for the coupling of the bag parameter to the scalar fields

$$
\left.B_{i}\left(\sigma, \sigma^{*}\right)=B_{0} \exp \left[-4\left(g_{i \sigma}^{\mathrm{bag}} \sigma+g_{i \sigma^{*}}^{\mathrm{bag}} \sigma^{*}\right) / M_{i}\right)\right]
$$

with $g_{i \sigma}^{\mathrm{bag}}$ and $g_{i \sigma^{*}}^{\mathrm{bag}}$ as additional parameters. Here it may be worth mentioning that in Ref. [18] the bag constant is coupled to the nonstrange $\sigma$ scalar field but not to $\sigma^{*}$.

The spurious center-of-mass energy is subtracted to obtain the effective baryon mass [35]

$$
M_{i}^{*}=\sqrt{E_{b a g}^{i}{ }^{2}-<p_{c m}^{2}>^{i}}
$$

where

$$
<p_{c m}^{2}>^{i}=\sum_{q}^{3} n_{q} x_{q}^{2} / R_{i}^{2}
$$

The bag radius $R_{i}$ for baryon species $i$ is obtained through the minimization of the baryon mass with respect to the bag radius 27]

$$
\frac{\partial M_{i}^{*}}{\partial R_{i}}=0
$$

The zero-point energy parameters $Z_{i}$ of Eq.(4) are used to fit the actual masses of the free baryons $M_{i}=939,1116,1189$ and $1315 \mathrm{MeV}$ and are found to be $Z_{i}=2.03,1.814,1.629$ and 1.505 for the $N, \Lambda, \Sigma$ and $\Xi$ hyperons respectively, corresponding to a free baryon bag parameter $B_{0}=(188.1)^{4} \mathrm{MeV}^{4}$ and a free nucleon bag radius $R_{0}=0.6 \mathrm{fm}$. 
The total energy density of cold infinite strange hadronic matter at finite baryon density $\rho_{B}$ is given by

$$
\varepsilon=\sum_{i} \frac{\gamma_{i}}{(2 \pi)^{3}} \int d^{3} k \sqrt{k^{2}+M_{i}^{* 2}} \theta\left(k_{F}^{i}-k\right)+\frac{1}{2} m_{\omega}^{2} \omega^{2}+\frac{1}{2} m_{\phi}^{2} \phi^{2}+\frac{1}{2} m_{\sigma}^{2} \sigma^{2}+\frac{1}{2} m_{\sigma^{*}}^{2} \sigma^{* 2}
$$

where the summation $i$ runs over the 8 members of the baryon octet which reduces for symmetric hypernuclear matter to 4 species with the spin-isospin degeneracy factors $\gamma_{i}=$ 4,2, 6 and 4 for $N, \Lambda, \Sigma$ and $\Xi$, respectively and where $\theta$ is the step function at the Fermi momentum $k_{F}^{i}$.

The effective Fermi energy of baryon species $i$ is given by

$$
\epsilon_{i}^{*}\left(k_{F}^{i}\right)=\sqrt{k_{F}^{i 2}+M_{i}^{* 2}}+g_{i \omega} \omega+g_{i \phi} \phi
$$

which is equal to the chemical potential $\mu_{i}$

$$
\mu_{i}=B_{i} \mu_{B}+S_{i} \mu_{S}
$$

where $B_{i}$ and $S_{i}$ are the baryon and strangeness quantum numbers, respectively. The two independent chemical potentials $\mu_{B}$ and $\mu_{S}$ are obtained from the conservation of the total baryon density

$$
\rho_{B}=\frac{1}{(2 \pi)^{3}} \sum_{i} B_{i} \gamma_{i} \int d^{3} k \theta\left(k_{F}^{i}-k\right)
$$

and the total strangeness density

$$
\rho_{S}=\frac{1}{(2 \pi)^{3}} \sum_{i} S_{i} \gamma_{i} \int d^{3} k \theta\left(k_{F}^{i}-k\right)
$$

The vector mean fields are determined by

$$
\omega=\sum_{i} \frac{g_{i \omega}}{m_{\omega}^{2}} \rho_{i}
$$

and

$$
\phi=\sum_{i} \frac{g_{i \phi}}{m_{\phi}^{2}} \rho_{i}
$$

where $g_{i \omega}$ and $g_{i \phi}$ are the meson-baryon coupling constants defined in Eqs. (18) and (19) below. by

The pressure is the negative of the grand thermodynamic potential density and is given

$$
P=\frac{1}{3} \sum_{i} \frac{\gamma_{i}}{(2 \pi)^{3}} \int d^{3} k \frac{k^{2}}{\epsilon_{i}^{*}} \theta\left(k_{F}^{i}-k\right)+\frac{1}{2} m_{\omega}^{2} \omega^{2}+\frac{1}{2} m_{\phi}^{2} \phi^{2}-\frac{1}{2} m_{\sigma}^{2} \sigma^{2}-\frac{1}{2} m_{\sigma^{*}}^{2} \sigma^{* 2} .
$$




\section{FITTING PARAMETERS FOR $Y Y$ POTENTIALS}

We assume that the $\sigma$ and $\omega$ mesons couple only to the up and down quarks while $\sigma^{*}$ and $\phi$ couple to the strange quark. We thus set $g_{r \phi}=g_{r \sigma^{*}}=g_{s \sigma}=g_{s \omega}=0$ where $r$ refers to the up and down flavors while $s$ denotes the strange flavor. By assuming the $S U(6)$ symmetry of the simple quark model we have the relations $g_{s \sigma^{*}}=\sqrt{2} g_{r \sigma}$ and $g_{s \phi}=\sqrt{2} g_{r \omega}$. The coupling of each baryon species with the vector mesons is calculated by counting the constituent quarks

$$
g_{i \omega}=\sum_{q}^{3} g_{q \omega}=\sum_{r} g_{r \omega}
$$

and

$$
g_{i \phi}=\sum_{q}^{3} g_{q \phi}=\sum_{s} g_{s \phi} .
$$

With these assumptions the only free parameters left at our disposal are the quark-meson coupling constants $g_{r \sigma}$ and $g_{r \omega}$ and the bag coupling constants $g_{i \sigma}^{\mathrm{bag}}, g_{i \sigma^{*}}^{\mathrm{bag}}$ for the 4 baryon species and these parameters are adjusted to fit nuclear properties as well as the extrapolated properties of hypernuclear matter. The coupling constants of the scalar and vector mesons to the nonstrange quarks are taken as $g_{r \sigma}=1$ and $g_{r \omega}=2.705$ which together with a bag coupling constant $g_{N \sigma^{*}}^{\mathrm{bag}}=6.81$ yield a binding energy of $16 \mathrm{MeV}$ and a compressibility $K_{V}^{-1}$ of $289 \mathrm{MeV}$ at the normal saturation density $\rho_{0}=0.17 \mathrm{fm}^{-3}$ of nuclear matter [29,31] .

Table I summarizes the values used in the current work for the basic quark-meson coupling constants as well as the two sets of the coupling constants $g_{i \sigma}^{\mathrm{bag}}$ and $g_{i \sigma^{*}}^{\mathrm{bag}}$ in the bag parameter $B_{i}\left(\sigma, \sigma^{*}\right)$. These sets are chosen to fit nuclear and hypernuclear properties. The parameters $g_{i \sigma}^{\mathrm{bag}}$ are taken to fit the hyperon potentials in nuclear matter:

$$
\begin{aligned}
& U_{\Lambda}^{(N)}\left(\rho_{0}\right)=-30 \mathrm{MeV}, \\
& U_{\Sigma}^{(N)}\left(\rho_{0}\right)=+30 \mathrm{MeV}, \\
& U_{\Xi}^{(N)}\left(\rho_{0}\right)=-18 \mathrm{MeV},
\end{aligned}
$$

where the hyperon potentials are defined by

$$
U_{i}^{(i)}=\left(M_{i}^{*}-M_{i}\right)+\left(g_{i \omega} \omega+g_{i \phi} \phi\right) .
$$

However, we make two different choices for the constants $g_{i \sigma^{*}}^{\mathrm{bag}}$. In the first set, referred to hereafter as MQMC-I, the medium constants $g_{i \sigma^{*}}^{\mathrm{bag}}$ are adjusted so that the potential of a single hyperon embedded in a bath of $\Xi$ matter becomes

$$
U_{\Xi}^{\Xi}\left(\rho_{0}\right)=U_{\Lambda}^{\Xi}\left(\rho_{0}\right)=-40 \mathrm{MeV}
$$

in accordance with the attractive hyperon-hyperon interaction of the Nijmegen potential Model D 13,18]. Furthermore, we adopt the approximation $U_{\Xi}^{\Xi}\left(\rho_{0}\right) \approx U_{\Sigma}^{\Xi}\left(\rho_{0}\right)$ to fit the medium constants. The resulting $U_{\Lambda}^{(\Lambda)}\left(\rho_{0} / 2\right)$ is about $-20 \mathrm{MeV}$. 
In MQMC-II, we adopt the $Y Y$ interactions which occur in the most recent $S U(3)$ extension of the Nijmegen soft-core potential Model NSC97 [20 22]. The phenomenology in this model departs substantially from that in MQMC-I. In particular, the $\Sigma \Sigma$ and $\Xi \Xi$ interactions are predicted to be highly attractive in some channels, leading to bound states. We have adjusted the bag constants $g_{i \sigma^{*}}^{\mathrm{bag}}$ where $i=\Lambda, \Sigma, \Xi$ to reproduce qualitatively the same binding energy curves of each hyperon species in its own hyperonic matter $B_{i}^{i}$ as those produced by the Model NSC97f [20,24]. The resulting binding energy curves are shown in Fig.11. No binding occurs for $\Lambda$ hyperons which are already unbound by $8-10 \mathrm{MeV}$ at the rather low density $\rho_{B}=0.05 \mathrm{fm}^{-3}$. On the other hand, $\Sigma$ matter is deeply bound at -33 $\mathrm{MeV}$ per baryon at $\rho_{\Sigma_{0}}$ which is twice as deep as ordinary nuclear matter, and $\Xi$ matter has an energy of $-23 \mathrm{MeV}$ per baryon at $\rho_{\Xi_{0}}$.

Furthermore, we have also considered the original (unmodified) QMC model where the bag constant is considered to be medium-independent and simply takes its free space value $B_{0}$. In analogy with the MQMC calculations we consider two sets of parameters. The QMC-I set reproduces the $Y Y$ potentials corresponding to the Nijmegen hard core potential model D as in MQMC-I. The scalar coupling constants are taken as $g_{q \sigma}=5.31$ and $g_{s \sigma^{*}}=$ $\sqrt{2} g_{q \sigma}$ while the vector ones are taken as $g_{q \omega}=1.471,1.868,5.148$ and 1.662 and $g_{s \phi}=0.0$, 2.289, 0.0 and 3.017 for $N, \Lambda, \Sigma$ and $\Xi$, respectively. The QMC-II set reproduces the $Y Y$ potentials corresponding to the Nijmegen soft-core potential NSC97 as in MQMC-II. The scalar coupling constants are taken to be the same as in QMC-I while the vector ones are taken as $g_{q \omega}=1.471,2.942,1.103$ and 1.721 and $g_{s \phi}=0.0,4.160,1.560$ and 2.434 for $N, \Lambda, \Sigma$ and $\Xi$, respectively.

\section{RESULTS AND DISCUSSIONS}

The resulting energy per particle curves of each baryon species $j$ in its own matter $E_{j}^{(j)}$ are depicted in Fig. 1 as a function of density for models MQMC-I and II. For nucleons, the curves are the same in both models since they fit the same nuclear matter properties. In MQMC-I, the interactions for $\Lambda$ and $\Sigma$ are repulsive with $E_{\Lambda}^{\Lambda}=+10 \mathrm{MeV}$ and $E_{\Sigma}^{\Sigma}=+5 \mathrm{MeV}$ at $\rho_{B}=0.15 \mathrm{fm}^{-3}$ for $\Lambda$ and $\Sigma$ hyperons respectively. The $\Lambda$ hyperon has a shallow local minimum at $\rho_{B}=0.13 \mathrm{fm}^{-3}$ while $E_{\Xi}^{\Xi}$ has an absolute minimum of $-7.5 \mathrm{MeV}$ per baryon at $\rho \cong 0.29 \mathrm{fm}^{-3}$. On the other hand, for MQMC-II, the energy $E_{\Lambda}^{\Lambda}$ reaches $+20 \mathrm{MeV}$ already at a density $\rho_{B}=0.1 \mathrm{fm}^{-3}$. This more repulsive potential can be attributed to the very weak underlying $\Lambda \Lambda$ interaction in the extended NSC97 Model. In contrast, the $\Sigma$ hyperon is deeply bound with a minimum of $-33 \mathrm{MeV}$ per baryon at $\rho_{B}=0.46 \mathrm{fm}^{-3}$. Also $\Xi$ hyperon matter has a strong attractive potential with a minimum of $-23 \mathrm{MeV}$ per baryon at $\rho=0.39 \mathrm{fm}^{-3}$. This shows that MQMC-II predicts that the $\Sigma \Sigma$ and $\Xi \Xi$ interactions are highly attractive in some channels, leading to bound states. It is also clear that a mixture of $\Sigma$ and $\Xi$ matter must be very deeply bound unless there is an overwhelmingly repulsive interaction between the $\Sigma$ and $\Xi$ hyperons.

Figs. 2 and 3 display the effective masses of the baryons $(N, \Lambda, \Sigma, \Xi)$ vs $\rho_{B}$ for several strangeness fractions $f_{S}$ using MQMC-I and II, respectively. In MQMC-I, the effective mass for each baryon species decreases monotonically with density. It is seen that as $f_{S}$ increases, $M_{N}^{*}$ increases while $M_{\Sigma}^{*}$ and $M_{\Xi}^{*}$ decrease. The $\Lambda$ hyperon effective mass however is only weakly sensitive to variations in $f_{S}$. In MQMC-II, the situation is rather different, as can 
be seen from Fig. 8. At first the effective masses of the nucleon and $\Lambda$ hyperon decrease smoothly with $\rho_{B}$ for low strangeness fractions. As $f_{S}$ exceeds 1.4, these effective masses jump suddenly to higher values as $\rho_{B}$ reaches a critical value and then start to decrease again monotonically for higher values of $\rho_{B}$. The jump or discontinuity in the effective mass of the nucleon can be as high as $75 \mathrm{MeV}$ for $f_{S}=1.5$ at a critical density of $0.57 \mathrm{fm}^{-3}$. The $\Lambda$ hyperon has a jump of around $40 \mathrm{MeV}$ for $f_{S}=1.5$ at a critical density $\rho_{B}=0.57 \mathrm{fm}^{-3}$. The effective masses of the $\Sigma$ and $\Xi$ hyperons have the opposite behavior with $M_{\Sigma}^{*}$ and $M_{\Xi}^{*}$ decreasing when the net strangeness fraction $f_{S}$ of the system increases. Furthermore they suddenly jump to lower values as $\rho_{B}$ exceeds a critical density for $f_{S}>1.4$. It is interesting to note that in MQMC-II the various effective baryon masses become independent of $f_{S}$ for $f_{S} \geq 1.5$ and $\rho_{B}>0.60 \mathrm{fm}^{-3}$. This behavior indicates that a phase transition takes place in MQMC-II at the critical densities where the discontinuities in the effective mass occur. The nature of this transition from the $N \Lambda \Xi$ phase to the $N \Sigma \Xi$ phase will become apparent in the following figures.

Figs. $⿴$ and 5 display the density dependence of the bag radius $R_{i}$ for the $N, \Lambda, \Sigma$ and $\Xi$ baryons for several strangeness fractions $f_{S}$ using models MQMC-I and II, respectively. The thick solid line indicates the limiting bag radius value $R_{\operatorname{exc}}=\left(3 / 4 \pi \rho_{B}\right)^{1 / 3}$ where the excluded volume occupies all space and the assumption of nonoverlapping bags definitely breaks down. It is interesting to note here that the bag radius in MQMC tends to increase with the increasing baryon density in contrary to the ordinary QMC models where the bag radius tends to decrease slowly or saturate at a constant value. This is because in MQMC the bag parameter decreases with density which allows the bag to expand. This problem can be cured by introducing quark-quark correlations [32] which however are not included in the present calculations. Such correlations tend to make the bags shrink at higher densities so that the overlap assumption is not violated and $R_{\text {exc }}$ will have values much larger than those given by the thick line in Fig. 6 . Finally it is observed that in MQMC-I the radius of the nucleon decreases while $R_{\Sigma}$ and $R_{\Xi}$ increase with increasing strangeness fractions while $R_{\Lambda}$ is almost unaffected by variations in $f_{S}$.

In contrast to MQMC-I where the bag radius increases monotonically with baryon density $\rho_{B}$ we notice from Fig. 5 that in MQMC-II this variation has sudden jumps or discontinuities for $f_{S} \geq 1.4$. For example, for a strangeness fraction $f_{S}=1.5, R_{N}$ drops from $0.775 \mathrm{fm}$ to $0.725 \mathrm{fm}$ when the baryon density reaches $\rho_{B}=0.55 \mathrm{fm}^{-3}$. The shrinking in the nucleon bag size for $f_{S}>1.4$ takes place before the breakdown in the nonoverlap assumption even in the current calculations where the quark-quark correlations are not included. Furthermore, the phase transition tends to occur at lower and lower densities as $f_{S}$ increases and the bag radius becomes independent of $f_{S}$. The same behavior is found for the radius of the $\Lambda$ hyperons but the situation is a quite different for the $\Sigma$ and $\Xi$ hyperons. $R_{\Sigma}\left(R_{\Xi}\right)$ both increase with respect to $f_{S}$ and both jump to a higher value at the critical baryon density when the phase transition takes place for $f_{S}$ exceeding 1.4. With $f_{S}=1.5, R_{\text {exc }}$ is reached at $\rho_{B}=0.50 \mathrm{fm}^{-3}$ for $\Sigma$ and $\Xi$ hyperons indicating that, even with the neglect of quarkquark correlations, MQMC-II is valid up to $\rho_{B}=0.50 \mathrm{fm}^{-3}$ for such large finite strangeness fractions.

Fig. 0 displays the binding energy per baryon for strange hadronic matter vs the baryon density $\rho_{B}$ for various strangeness fractions $f_{S}$. It is noted in Fig.6(a) that the energy curves for MQMC-I have only one global minimum for every value of the strangeness fraction $f_{S}$. 
At first, the depth of the minimum increases as $f_{S}$ increases until it reaches a strangeness fraction of about 1.3 after which the depth of the minimum decreases again for higher values of $f_{S}$ and approaches that of purely $\Xi$ matter for $f_{S}=2$. It is found that in MQMC-I strange hadronic matter is built out always from a mixture of $N, \Lambda$ and $\Xi$ baryons. Hence each energy curve has only one global minimum.

Fig. 6(b) indicates that the situation for MQMC-II is drastically different. For low values of $f_{S}$ each curve still has a single minimum whose depth increases with the increase in the strangeness fraction. However, when $f_{S}$ reaches about 1.3 , a second minimum appears at a rather high density $\rho_{B}=0.86 \mathrm{fm}^{-3}$. Moreover, this second energy minimum quickly deepens and reaches -39 and $-56 \mathrm{MeV}$ for $f_{S}=1.4$ and 1.5 , respectively. The appearance of this second minimum can be attributed to the increasingly dominant presence of the $\Sigma$ hyperons in the system which seems to undergo a first order phase transition from $N \Lambda \Xi$ and $N \Xi$ to $N \Sigma \Xi$ strange hadronic matter but at a rather higher baryonic density. For values of the strangeness fraction $f_{S}$ greater than 1.5 the potential well depth starts to decrease again as the $\Xi$ starts to dominate and the energy curves gradually approach that for pure $\Xi$ matter for $f_{S}=2$.

Fig. 7 displays the depth of the energy minimum and the corresponding baryonic density as functions of the strangeness fraction $f_{S}$. It is evident that the depth of the minimum in MQMC-I increases with the strangeness fraction until the curve has a maximum binding energy of $19 \mathrm{MeV}$ at $f_{S}=1.2$. The binding energy magnitude decreases again as $f_{S}$ increases to form pure $\Xi$ matter at $f_{S}=2.0$ with a binding energy of $10 \mathrm{MeV}$. As can be seen from Fig.7(b) the locations of these minima appear at higher densities as $f_{S}$ increases until it reaches $f_{S}=1.2$ where it hovers around $\rho_{B}=0.40 \mathrm{fm}^{-3}$ for $f_{S}=1.2-1.6$. Beyond $f_{S}=1.6$, the location of the energy minimum moves to lower densities.

The situation for MQMC-II is rather similar to that of MQMC-I for $f_{S}<1.3$, but it is essentially different for $f_{S} \geq 1.3$. When the strangeness fraction reaches $f_{S}=1.3$, a second minimum in the binding energy appears at $\rho_{B}=0.86 \mathrm{fm}^{-3}$. Furthermore, the first minimum disappears at $f_{S}=1.5$ and a transition to the second minimum (the $N \Sigma \Xi$ phase) occurs due to the reaction $N \Xi \rightarrow \Sigma \Sigma$. The upper and lower branches of the MQMC-II curve in Fig. I(b) thus refer to two different phases with the discontinuity in the density of the system indicating it is a first order transition. The binding energy reaches a maximum of $56 \mathrm{MeV}$ for $f_{S}=1.5$ and $\rho_{B}=0.64 \mathrm{fm}^{-3}$ and then decreases to $25.7 \mathrm{MeV}$ as $f_{S}$ increases to $f_{S}=2$ where the second minimum disappears since the system is now composed solely of $\Xi$ hyperons. We also display in Fig. 7 the results for the QMC-I and QMC-II models which are seen to be very similar to those found in MQMC-I with a single stable $N \Lambda \Xi$ phase. In particular, unlike MQMC-II, QMC-II does not lead to a stable $N \Sigma \Xi$ system. Finally it is worth mentioning that, due to the shrinking of the bag radius with increasing baryon density, the nonoverlap assumption survives up to $\rho_{B}>1.1 \mathrm{fm}^{-3}$.

Figs. 8 and 9 display the fractional density of each baryon species $\rho_{i} / \rho_{B}$ in strange hadronic matter versus the total baryon density $\rho_{B}$ for several values of $f_{S}$ for Models I and II, respectively. It is seen that in MQMC-I the $\Sigma$ hyperon does not appear at all while the $\Lambda$ has a substantial contribution for low densities $\rho_{B} \leq 0.1 \mathrm{fm}^{-3}$. The $\Lambda$ contribution, however, drops substantially for higher densities to saturate at a constant value around $\rho_{\Lambda} / \rho_{B}=0.20-0.25$ for $\rho_{B} \geq 0.2 \mathrm{fm}^{-3}$ and $f_{S}=0.5-1.8$. It also disappears for $f_{S}=0$ (nuclear matter) and $f_{S}=2.0$ (pure $\Xi$ matter). The contribution of the nucleons $\rho_{N} / \rho_{B}$ 
decreases monotonically from one to zero as $f_{S}$ increases from zero to two. For $f_{S} \geq 1$ the nucleons comprise a surprisingly small fraction of the total number of baryons for small densities but their contribution increases rapidly and saturates at higher densities. The variation of the contribution of the $\Xi$ hyperons with density is similar to that of the nucleons but with $\rho_{\Xi} / \rho_{B}$ increasing from zero to one as $f_{S}$ increases from zero to two.

Fig. 9 shows however that the situation is significantly different in MQMC-II, with the $\Sigma$ hyperons appearing above some critical density and their contribution rapidly saturating at higher densities. The fractional density $\rho_{\Sigma} / \rho_{B}$ takes its maximum value of about $50 \%$ at $f_{S}=1.5$. The $\Lambda$ hyperon contribution is large at low baryon densities $\rho_{B}<0.10$ but practically disappears for higher densities for $f_{S} \geq 1$. The fractional densities for the $N$ 's and $\Xi$ 's in MQMC-II are similar to those in MQMC-I except for $f_{S} \geq 1.2$ where there is a depletion at high densities caused by the appearance of the $\Sigma$ hyperons in the system. The nucleons even disappear completely at high densities for $f_{S} \geq 1.5$.

Figs. 10 and 11 display $\mu_{i} / \epsilon_{i}^{*}(0)$ vs $\rho_{B} \epsilon_{i}^{*}$ is the Fermi energy and $\mu_{i}$ is the chemical potential, eqs. (11) and (12). If the ratio $\mu_{i} / \epsilon_{i}^{*}\left(k_{F}=0\right)<0$ baryons of species $i$ cannot exist in the system. The baryon species first appears with zero fermi momentum $k_{F}=0$ when this ratio reaches one and then takes a finite fermi momentum as the ratio exceeds one. ¿From Fig. 10 it is evident that in MQMC-I the $\Sigma$ hyperons cannot be produced at all in strange hadronic matter. Furthermore, $N$ production is increasingly suppressed while $\Xi$ production is enhanced as $f_{S}$ increases.

The situation is quite different in MQMC-II, Fig. 11, where the $\Sigma$ hyperons appear in the system for strangeness fractions $f_{S} \geq 1.0$. They first appear at $\rho_{B} \geq 0.85$ for $f_{S}=1.0$ and as $f_{S}$ increases they appear at lower baryon densities. However, the situation is opposite for $\Lambda$ which is produced abundantly for low strangeness fractions $f_{S}<1.0$ and then disappears when $f_{S}$ exceeds 1.0. The contribution of the nucleons decreases significantly for $f_{S}>1.3$ and they disappear completely from the system at $f_{S}=1.5$ when the baryon density exceeds $\rho_{B}=0.65 \mathrm{fm}^{-3}$. Furthermore, the nucleons disappear at lower baryon densities as the strangness fraction $f_{S}$ increases. It is thus seen that $N$ production in MQMC-II is influenced drastically by the transition from the $N \Lambda \Xi$ phase to the $N \Sigma \Xi$ phase. On the other hand, $\Xi$ hyperon production in MQMC-II is not much different from that in MQMC-I.

Finally, Fig.12 displays the pressure vs the baryon density $\rho_{B}$ for Models I and II. MQMCI has the normal van der Waal-type loop at small densities reflecting the gas to liquid phase transition. In MQMC-II the pressure has an additional sudden and significant drop at high densities $\rho_{B}>0.55 \mathrm{fm}^{-3}$ for strangeness fractions $f_{S} \geq 1.5$. This dramatic drop in the pressure is due to the transition from the $N \Lambda \Xi$ phase to the $N \Sigma \Xi$ phase in strange hadronic matter and probably provides an experimental clue for it.

\section{SUMMARY AND CONCLUSION}

In the present work, we have studied, within the context of the MQMC model, the properties of strange hadronic matter composed of the $\mathrm{SU}(3)$ octet baryons $N, \Lambda, \Sigma$ and $\Xi$ using two different models for the hyperon-hyperon interactions. It is interesting to note here that the medium dependent bag constant for each baryon species reflect more realistic

self interaction with the scalar mean fields which are corresponding to the nonlinear terms of the scalar mean fields in the normal RMF calculations. 
MQMC-I is designed to mimic the consequences of the Nijmegen hard-core potential model D 19, 13, 17]. It is nevertheless constrained by $\Lambda$ and $\Xi$ nuclear phenomenology, and by a few $\Lambda \Lambda$ hypernuclear species reported to date. It accounts more realistically for the attractive $\Lambda \Lambda$ and $N \Xi$ interactions, but ignores altogether $\Sigma$ hyperons. On the other hand, MQMC-II is designed to generate qualitatively similar baryon potentials to those obtained in the BHF approximation from the SU(3) extensions of the Nijmegen soft-core NSC97 potential models. The NSC97 model has been tuned up to reproduce certain characteristics of $\Lambda$ hypernuclei, particularly its version NSC97f. It yields particularly attractive $\Xi \Xi, \Sigma \Sigma$ and $\Sigma \Xi$ interactions.

In MQMC-I, the $\Sigma$ hyperons do not appear at all and the $\Lambda$ hyperons have a substantial contribution at low densities for medium strangeness fractions $f_{S}=0.5-1.8$. However this contribution drops quickly and saturates for $\rho_{B} / \rho_{0} \geq 1$ at a constant fraction density slightly larger than $20 \%$. This model produces smooth binding energy curves over the entire range of $f_{S}$ and $\rho_{B}$ and forms (meta-)stable strange hadronic matter in the bulk.

Due to the exceptionally strong attractive interactions among the $\Sigma$ and $\Xi$ hyperons in MQMC-II, the $N \Lambda \Xi$ - and $N \Xi$-dominated strange hadronic matter that exists for $f_{S} \leq 1.3$ is replaced by $N \Sigma \Xi$-dominated matter for $f_{S} \geq 1.3$, with binding energies per baryon reaching as much as $56 \mathrm{MeV}$ for $f_{S}=1.5$. The equation of state becomes much softer and seems to allow deeply bound hyperonic matter. The system built out of $N \Sigma \Xi$ forms a particularly (meta-)stable state of strange hadronic matter in the bulk. When the first order phase transition takes place above some critical baryon density and strangeness fraction, the $\Sigma$ hyperons appear and their contribution saturates rapidly to a constant fraction density that takes its maximum value of about $50 \%$ at $f_{S}=1.5$. It is expected that such a phase transition may drastically change the global features of neutron stars [36].

Our results for MQMC-II are qualitatively similar to those of Schaffner-Bielich and Gal [24] where the same $Y Y$ potentials of MQMC-II (what they call Model N) are employed within RMF theory but with only hadronic degrees of freedom. There are however some important quantitative differences. Whereas in Ref. [24] it is found that the second energy minimum, corresponding to $N \Sigma \Xi$ dominated matter, becomes more stable than the first minimum for $f_{S} \geq 1$, our current results indicate that this takes place at higher strangeness fractions $f_{S} \geq 1.3$. Furthermore, the binding energy well for the $N \Sigma \Xi$ system has a maximum depth of $56 \mathrm{MeV}$ in the present work while Ref. [24] finds the largest depth to be 78 $\mathrm{MeV}$. In both calculations the largest binding for the $N \Sigma \Xi$ system takes place at $f_{S} \approx 1.5$. The fact that the phase transition takes place at larger strangeness fractions may make it more difficult to observe experimentally, if it occurs at all.

Finally, quark-quark correlations and excluded volume effects may become important at high baryon densities in particular when the bags start to overlap at baryon densities $\rho_{B}=2-3 \rho_{0}$. We shall consider these effects on the properties of strange hadronic matter in our future work.

\section{ACKNOWLEDGMENTS}

Financial support by the Deutsche Forschungsgemeinschaft through grant GR 243/51-2 is gratefully acknowledged. We also thank A. Gal for helpful discussions. 


\section{REFERENCES}

[1] A. R. Bodmer, Phys. Rev D 4, 1601 (1971).

[2] E. Witten, Phys. Rev. D 30, 272 (1984).

[3] E. Farhi and R. L. Jaffe, Phys. Rev. D 30, 2379 (1984).

[4] M. S. Berger and R. L. Jaffe, Phys. Rev. C 35,213 (1987)

[5] E. P. Gilson and R. J. Jaffe, Phys. Rev. Lett. 71, 332 (1993).

[6] C. Greiner and H. Stöcker, Phys. Rev. D 44, 3517 (1991)

[7] H. W. Barz, B. Friman, J. Knoll and H. Schulz, Phys. Lett. B242, 328 (1990).

[8] J. Schaffner-Bielich, Nucl. Phys. A639, 443c (1998).

[9] J. L. Nagle for the E864 Collaboration, Nucl. Phys. A661, 185c (1999).

[10] J. Schaffner, C. Greiner and H. Stoöcker, Phys. Rev. C 46, 322 (1992).

[11] K. S. Lee and U. Heinz, Phys. Rev. D 47, 2068 (1993).

[12] N. Glendenning, Phys. Rev. C 23, 2757 (1981).

[13] J. Schaffner, C. B. Dover, A. Gal, D. J. Millener, C. Greiner and H. Stöcker, Ann. Phys. (N.Y.) 235, 35 (1994).

[14] J. Schaffner, C. B. Dover, A. Gal, C. Greiner, and H. Stöcker, Phys. Rev. Lett. 71, 1328 (1993).

[15] K. Ikeda, H. Bando, and T. Motoba, Prog. Theor. Phys. Suppl. 81, 147 (1985).

[16] H.-J. Schulze, M. Baldo, U. Lambardo, J. Cugnon, and A. Lejeune, Phys. Rev. C 57 704 (1998).

[17] S. Balberg, A. Gal and J. Schaffner, Prog. Theor. Phys. Suppl. 117, 325 (1994).

[18] P. Wang, R. K. Su, H.Q. Song, and L.L. Zhang, Nucl. Phys. A653, 166 (1999).

[19] M.M. Nagels, T.A. Rijken, and J.J. de Swart, Phys. Rev. D 15, 2547 (1977).

[20] V. G. J. Stoks and T.S.H. Lee, Phys. Rev. C 60, 024006 (1999)

[21] V. G. J. Stoks and Th. A. Rijken, Phys. Rev. C 59, 3009 (1999).

[22] Th. A. Rijken, V. G. J. Stoks, and Y. Yamamoto, Phys. Rev. C 59, 21 (1999).

[23] I. Vidaña, A Polls, A. Ramos, M. Hjorth-Jensen, and V.G.J. Stoks, Phys. Rev. C 61, $025802(2000)$.

[24] J. Schaffner-Bielich and A. Gal, Phys. Rev C 62034311 (2000).

[25] P. A. M. Guichon, Phys. Lett. B 200, 235 (1988).

[26] P. G. Blunden and G. A. Miller, Phys. Rev. C 54, 359 (1996).

[27] K. Saito and A. W. Thomas, Phys. Lett. B 327, 9 (1994).

[28] X. Jin and B. K. Jennings, Phys. Rev. C 54, 1427 (1996); Phys. Lett. B 374, 13 (1996).

[29] X. Jin and B. K. Jennings, Phys. Rev. C 55, 1567 (1997); H. Müller and B. K. Jennings, Nucl. Phys. A 626, 966 (1997); H. Müller, Phys. Rev. C 57, 1974 (1998).

[30] M. K. Banerjee, Phys. Rev. C 45, 1357 (1992); V. K. Mishra, Phys. Rev. C 46, 1143 (1992); E. Naar and M. C. Birse, J. Phys. G. 19, 555 (1993)

[31] I. Zakout and H. Jaqaman, Phys.Rev. C 59962 (1999);ibid 59968 (1999).

[32] I. Zakout and H. R. Jaqaman, J.Phys. G 261095 (2000).

[33] I. Zakout, H. R. Jaqaman, S. Pal, H. Stöcker and W. Greiner, Phys. Rev. C 61055208 (2000).

[34] S. Pal, M. Hanauske, I. Zakout, H. Stöcker and W. Greiner Phys. Rev. C 60015802 (1999).

[35] S. Fleck, W. Bentz, K. Shimizu, and K. Yazaki, Nucl. Phys. A510, 731 (1990).

[36] J. Schaffner-Bielich, M. Hanauske, H. Stöcker and W. Greiner, astro-ph/0005490. 


\section{TABLES}

TABLE I. Fitting parameters

\begin{tabular}{cccccccccc}
\hline \hline Fit set & $g_{q \sigma}$ & $g_{q \omega}$ & $g_{N \sigma}^{\text {bag }}$ & $g_{\Lambda \sigma}^{\text {bag }}$ & $g_{\Lambda \sigma^{*}}^{\text {bag }}$ & $g_{\Sigma \sigma}^{\text {bag }}$ & $g_{\Sigma \sigma^{*}}^{\text {bag }}$ & $g_{\Xi \sigma}^{\text {bag }}$ & $g_{\Xi \sigma^{*}}^{\text {bag }}$ \\
\hline MQMC-I & 1.0 & 2.705 & 6.81 & 4.22 & 5.45 & 1.63 & 7.26 & 2.27 & 9.12 \\
MQMC-II & 1.0 & 2.705 & 6.81 & 4.22 & 0.0 & 1.63 & 10.28 & 2.27 & 10.17 \\
\hline \hline
\end{tabular}




\section{FIGURES}

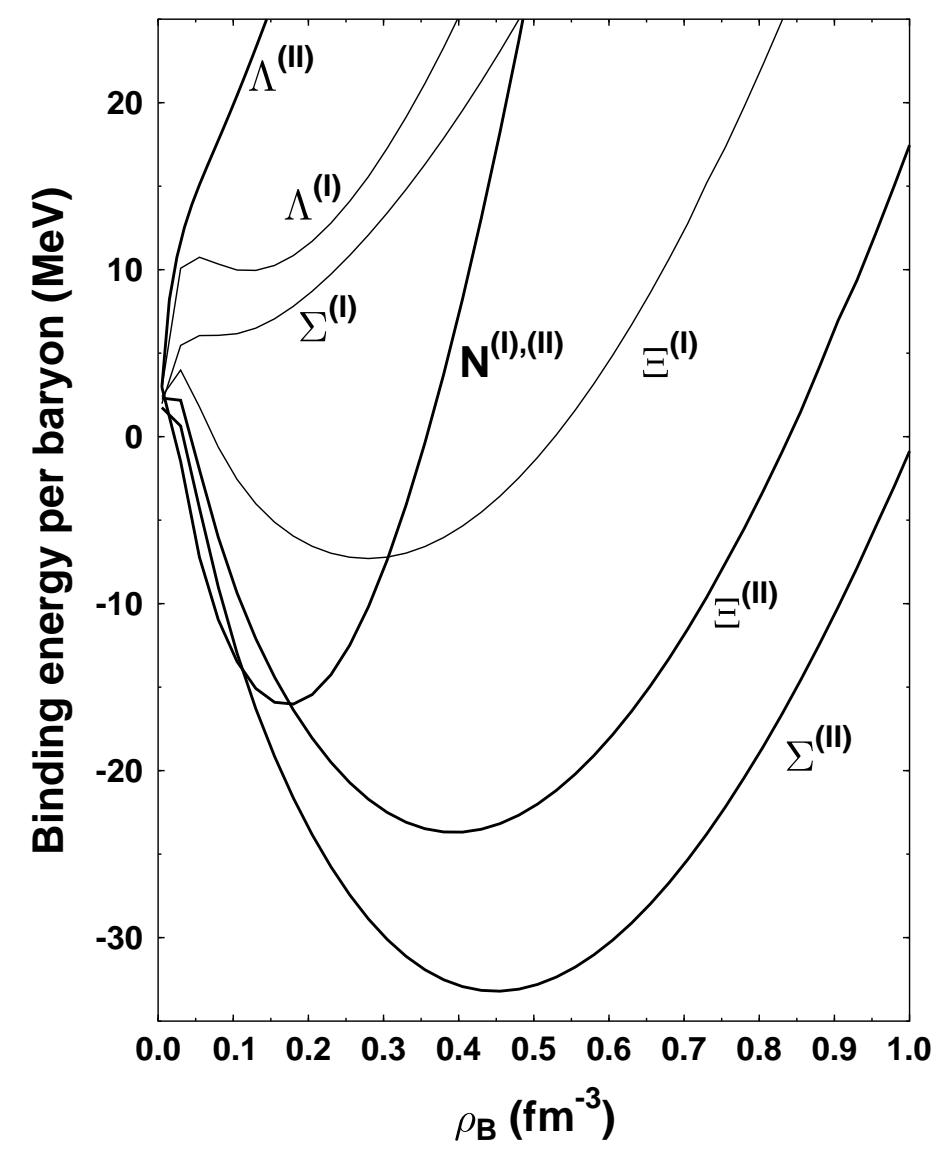

FIG. 1. Binding energy per nucleon $(N)$ in nuclear matter, compared to the binding energy per hyperon $(\Lambda, \Sigma, \Xi)$ in its own hyperonic matter. The hyperonic parameters where chosen to fit MQMC-I and II as discussed in the text. 

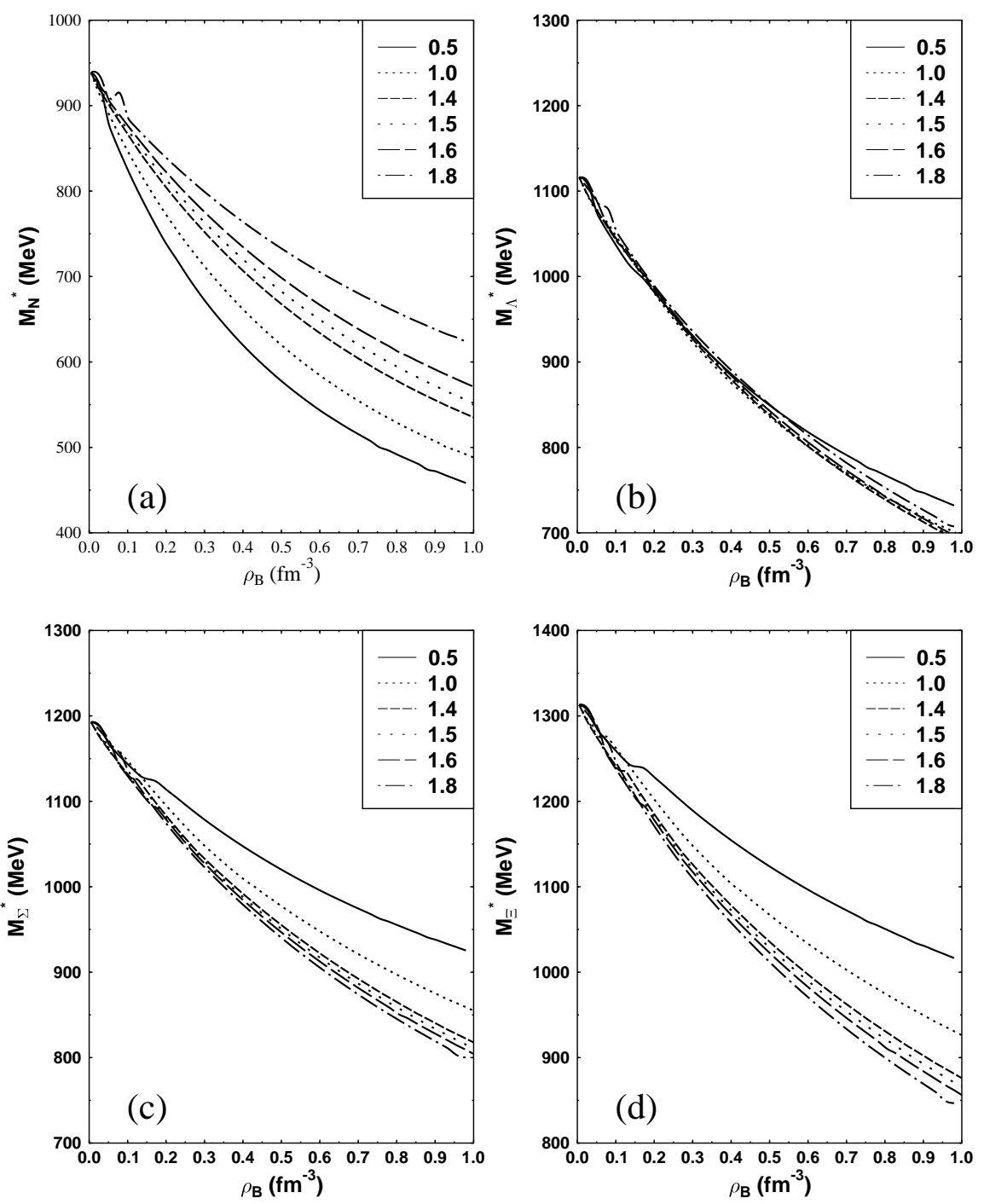

FIG. 2. The effective mass vs baryon density in MQMC-I for the $N, \Lambda, \Sigma, \Xi$ baryons. 

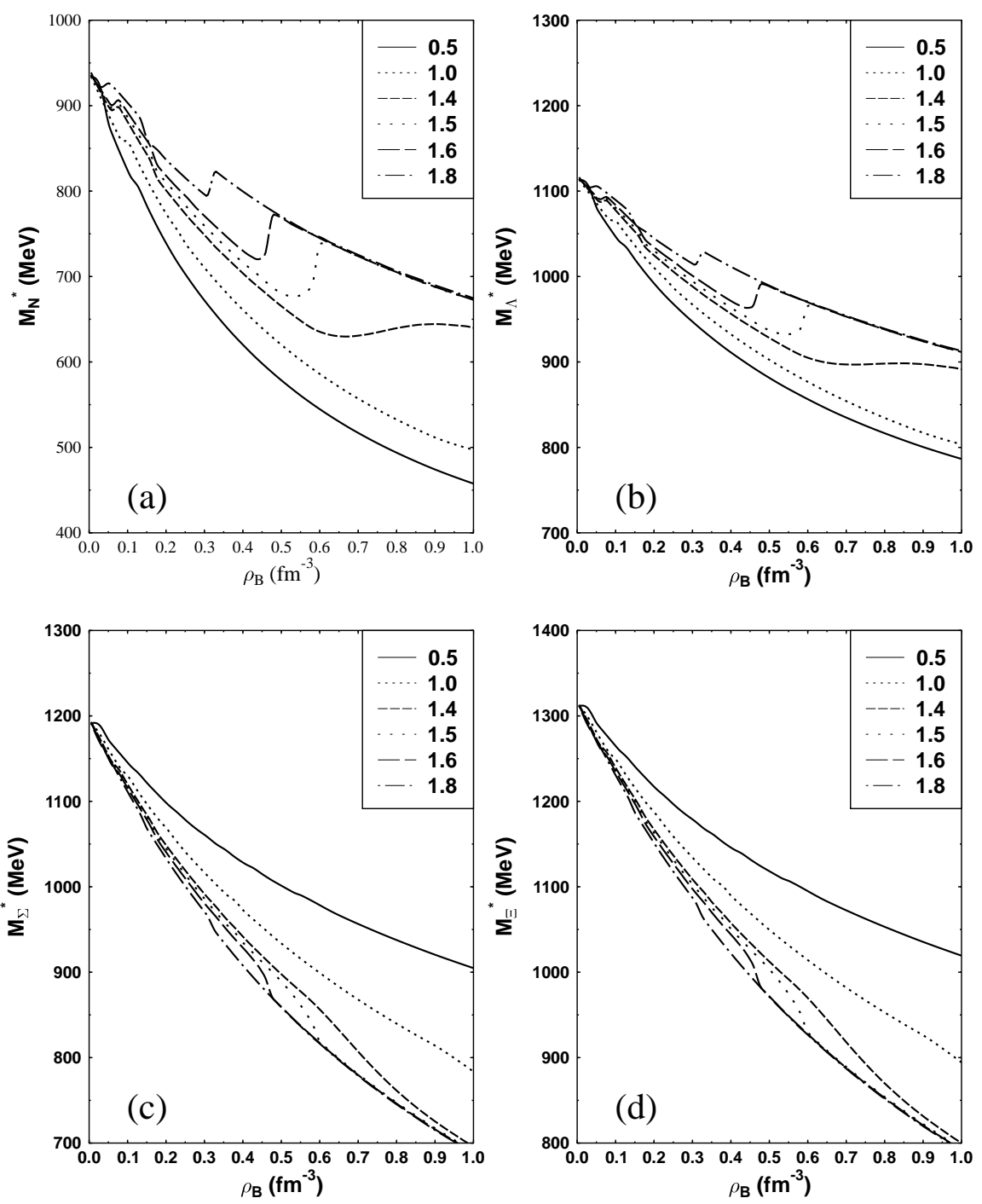

FIG. 3. The effective mass vs baryon density in MQMC-II for the $N, \Lambda, \Sigma, \Xi$ baryons. 

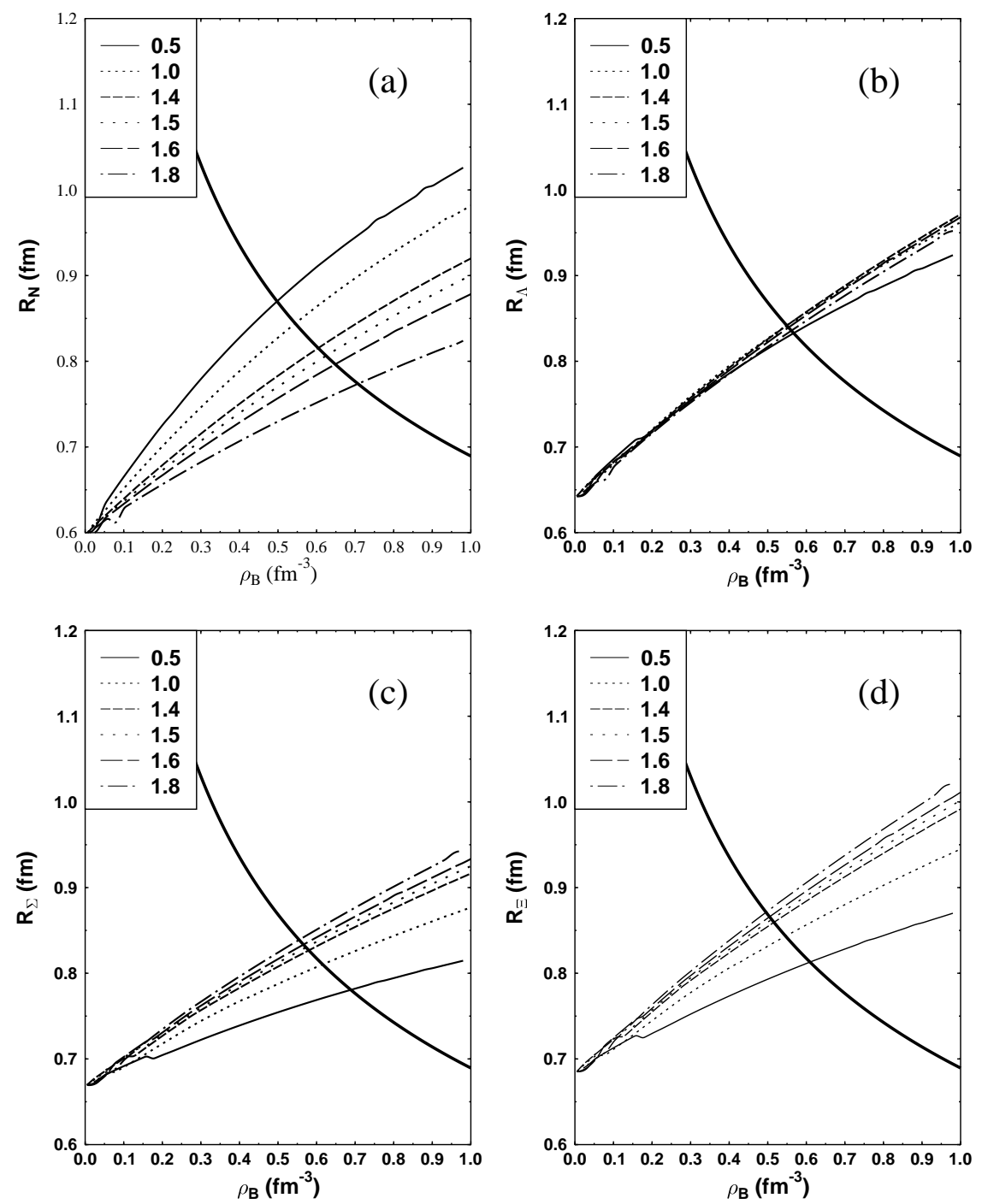

FIG. 4. The bag radius vs baryon density in MQMC-I for the $N, \Lambda, \Sigma, \Xi$ baryons. 

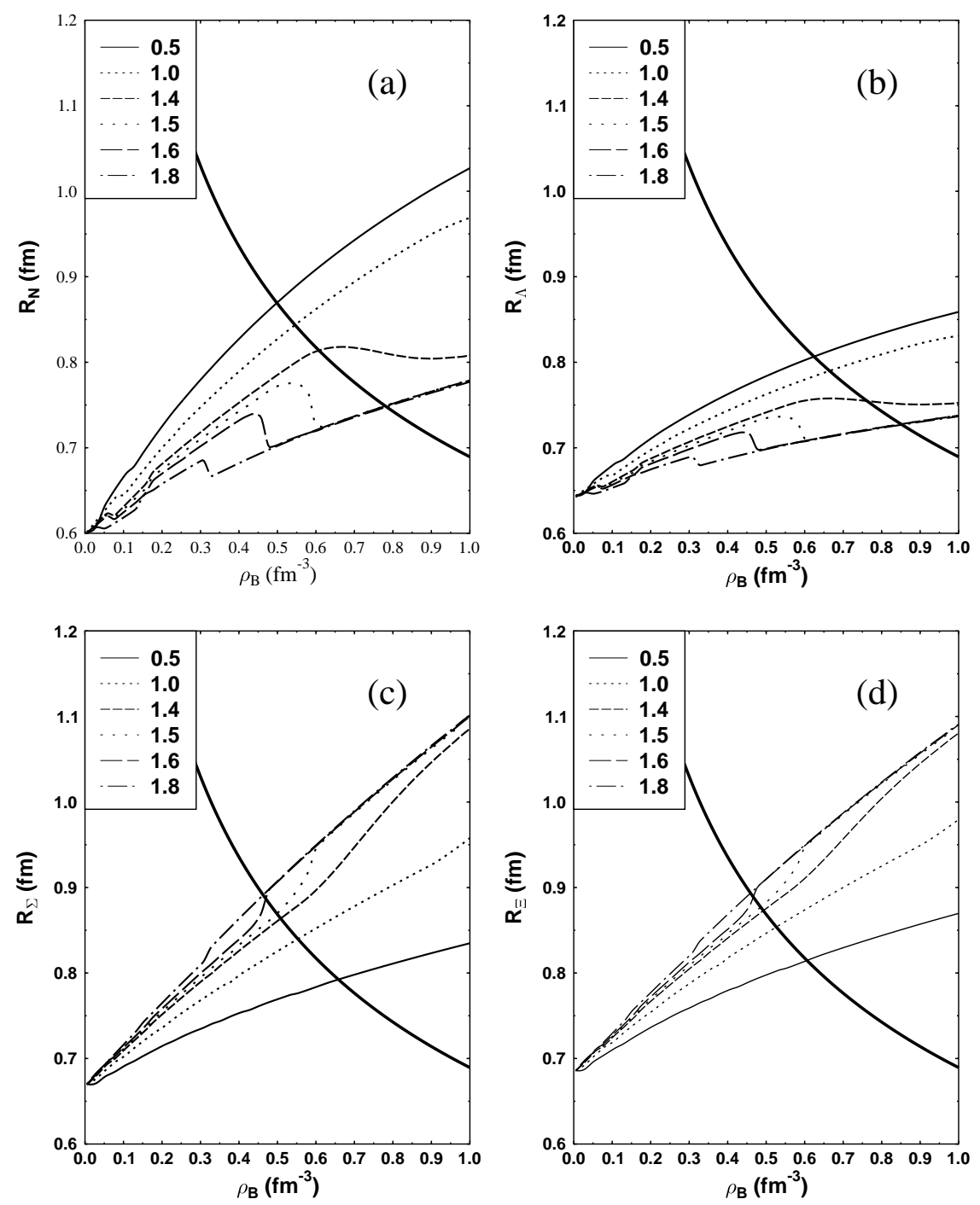

FIG. 5. The bag radius vs baryon density in MQMC-II for the $N, \Lambda, \Sigma, \Xi$ baryons. 

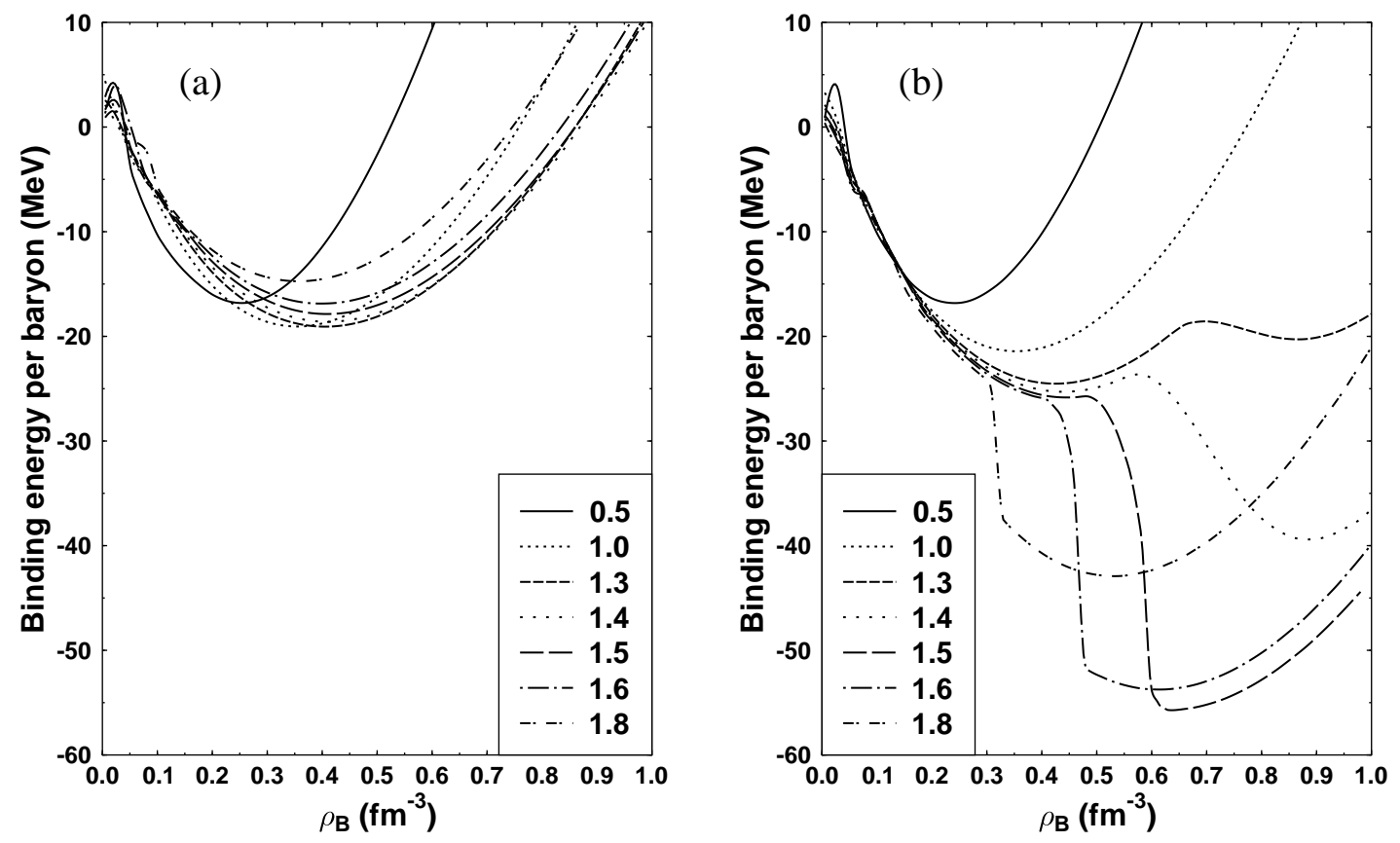

FIG. 6. The binding energy per baryon of strange hadronic matter for various strangeness fractions in (a) MQMC-I and (b) MQMC-II. 

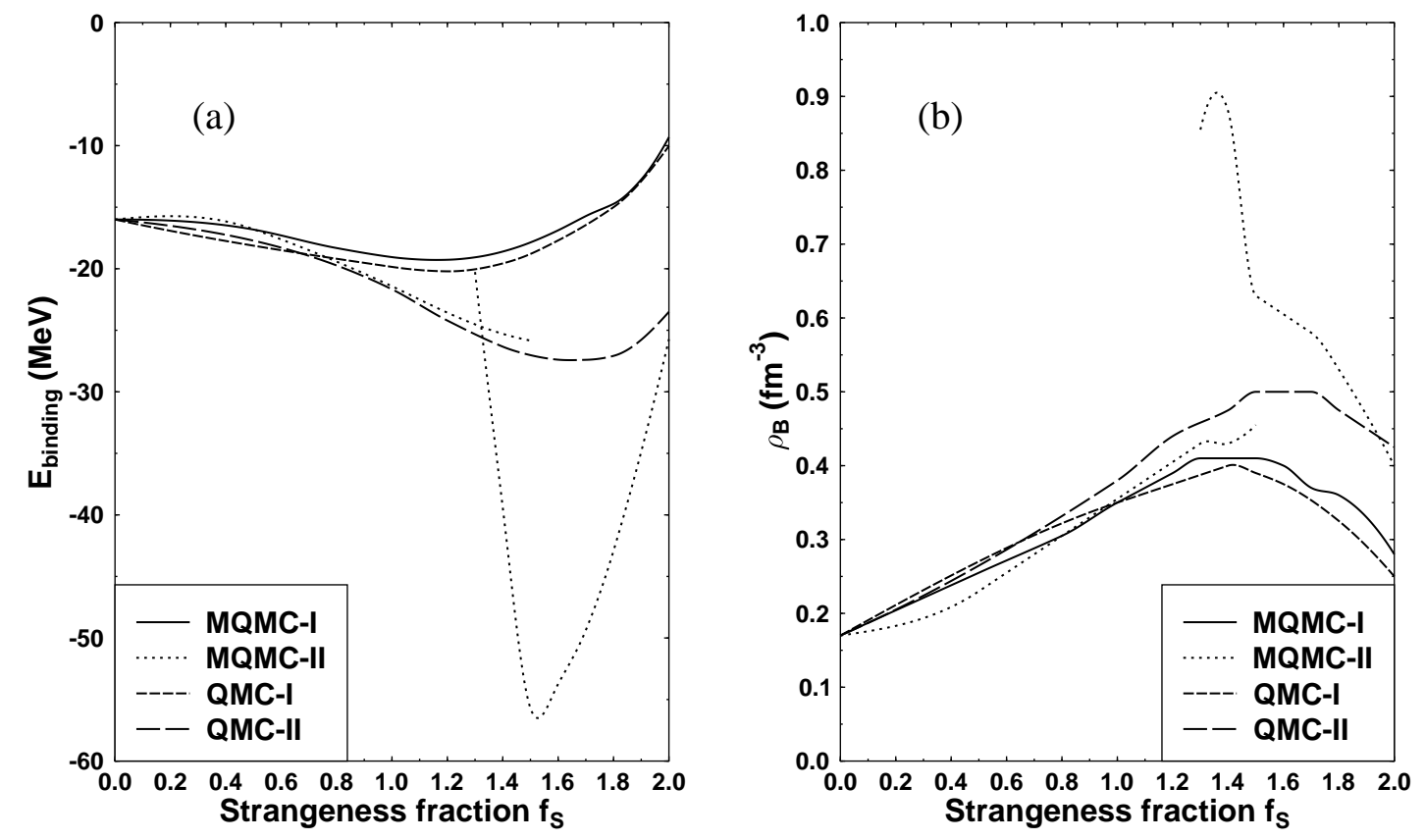

FIG. 7. Comparison of (a) the depth and (b) the location of the energy minimum of strange hadronic matter as functions of the strangeness fraction $f_{s}$ in MQMC-I and MQMC-II as well as in QMC-I and QMC-II. 

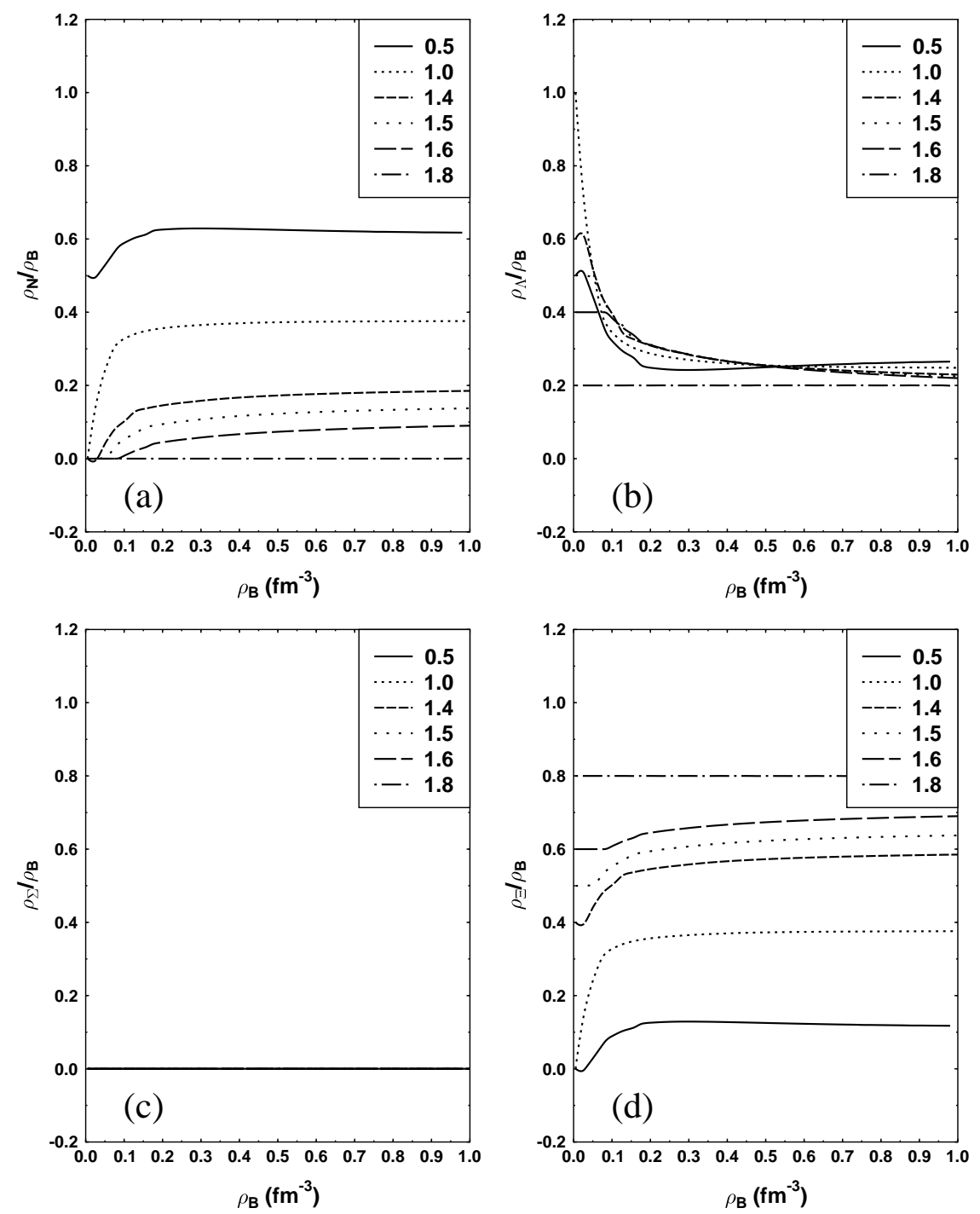

FIG. 8. Fractional density $\rho_{i} / \rho_{B}$ vs. $\rho_{B}$ for each baryon species in MQMC-I with different strangeness fractions. (a) $N$, (b) $\Lambda$, (c) $\Sigma$ and (d) $\Xi$. 

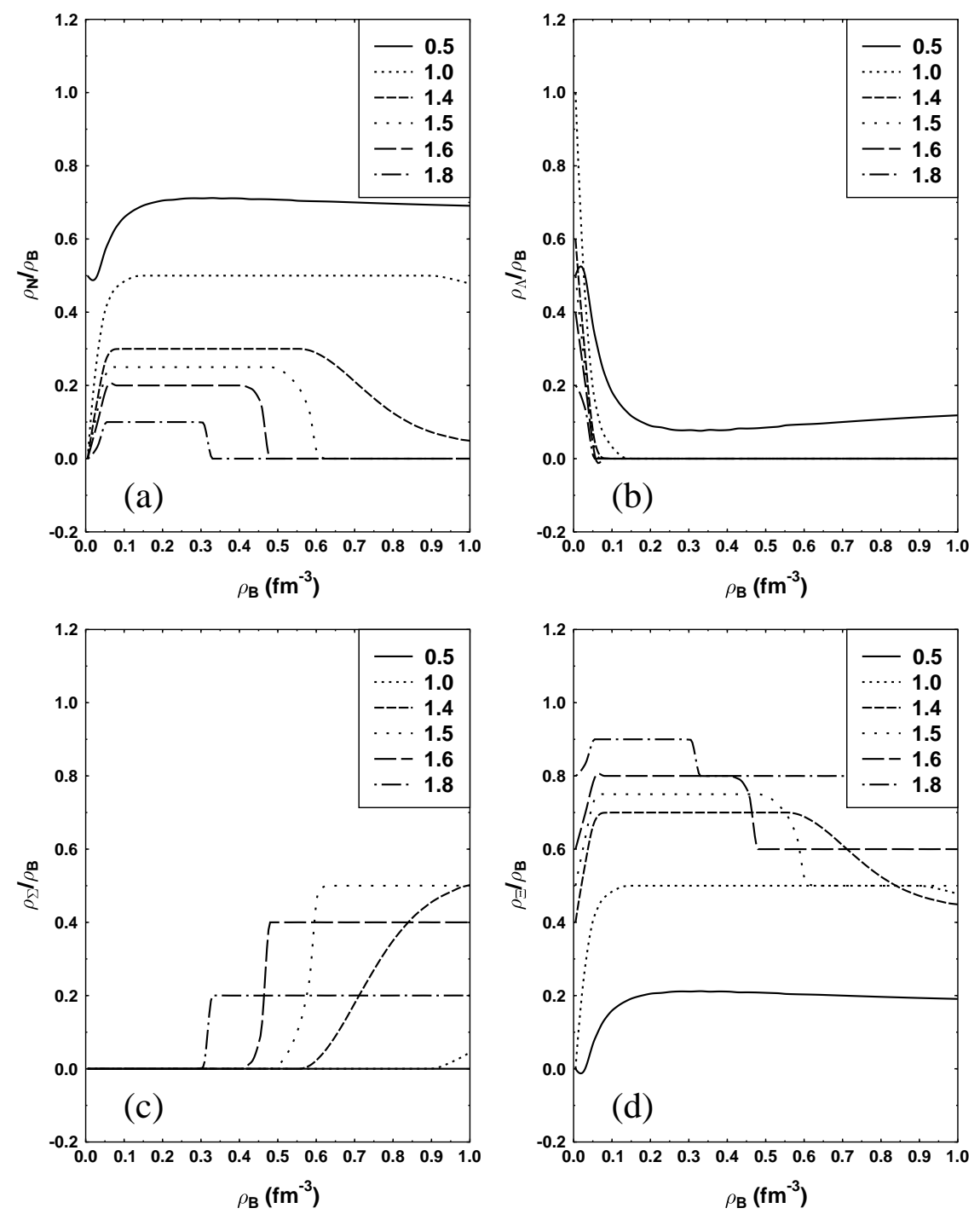

FIG. 9. Fractional density $\rho_{i} / \rho_{B}$ vs. $\rho_{B}$ for each baryon species in MQMC-II with different strangeness fractions. (a) $N$, (b) $\Lambda$, (c) $\Sigma$ and (d) $\Xi$. 

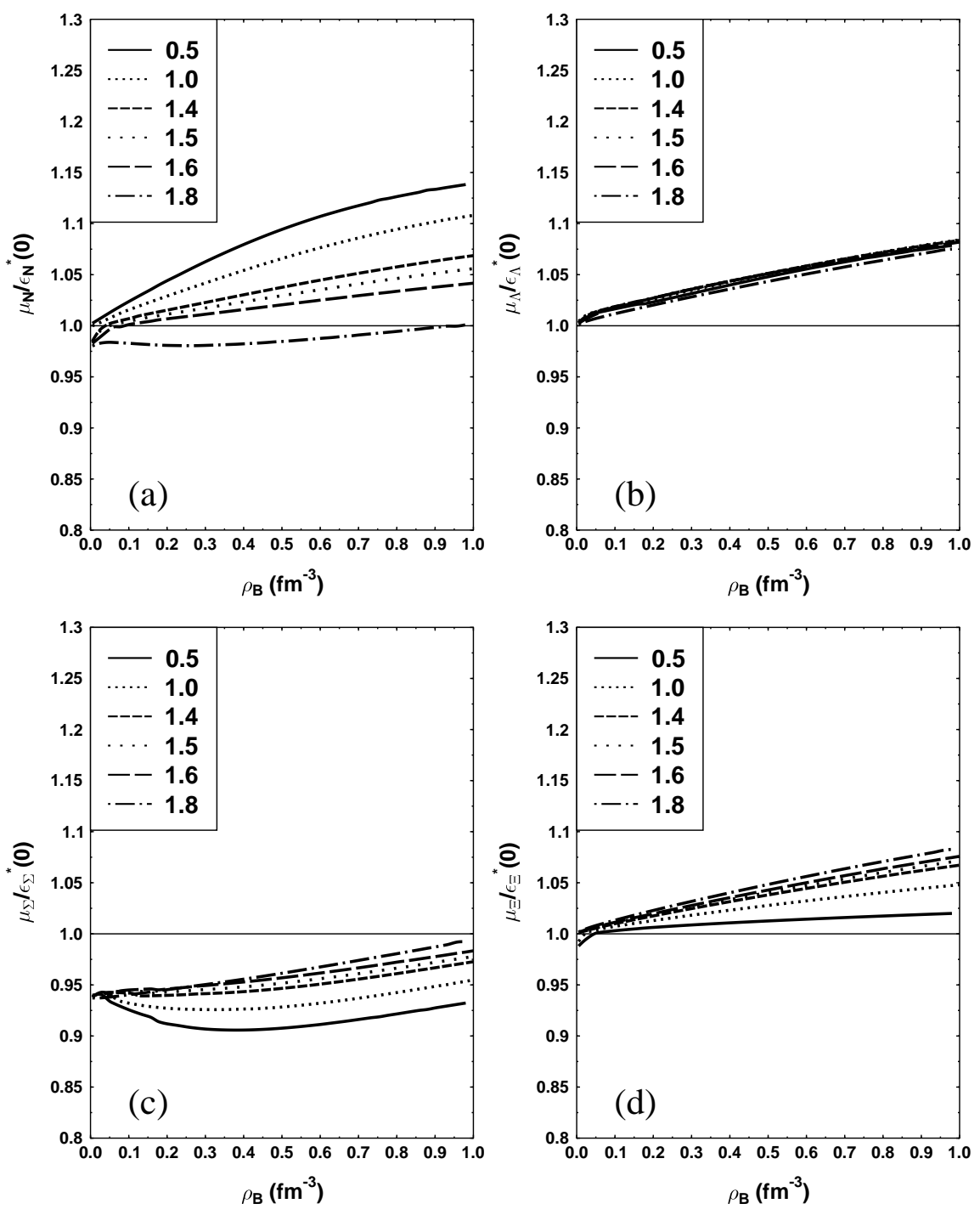

FIG. 10. The threshold production ratio $\mu_{i} / \epsilon_{i}^{*}\left(k_{F}=0\right)$ for each baryon species and for different strangeness fractions in MQMC-I. (a) $N$, (b) $\Lambda$, (c) $\Sigma$ and (d) $\Xi$. 

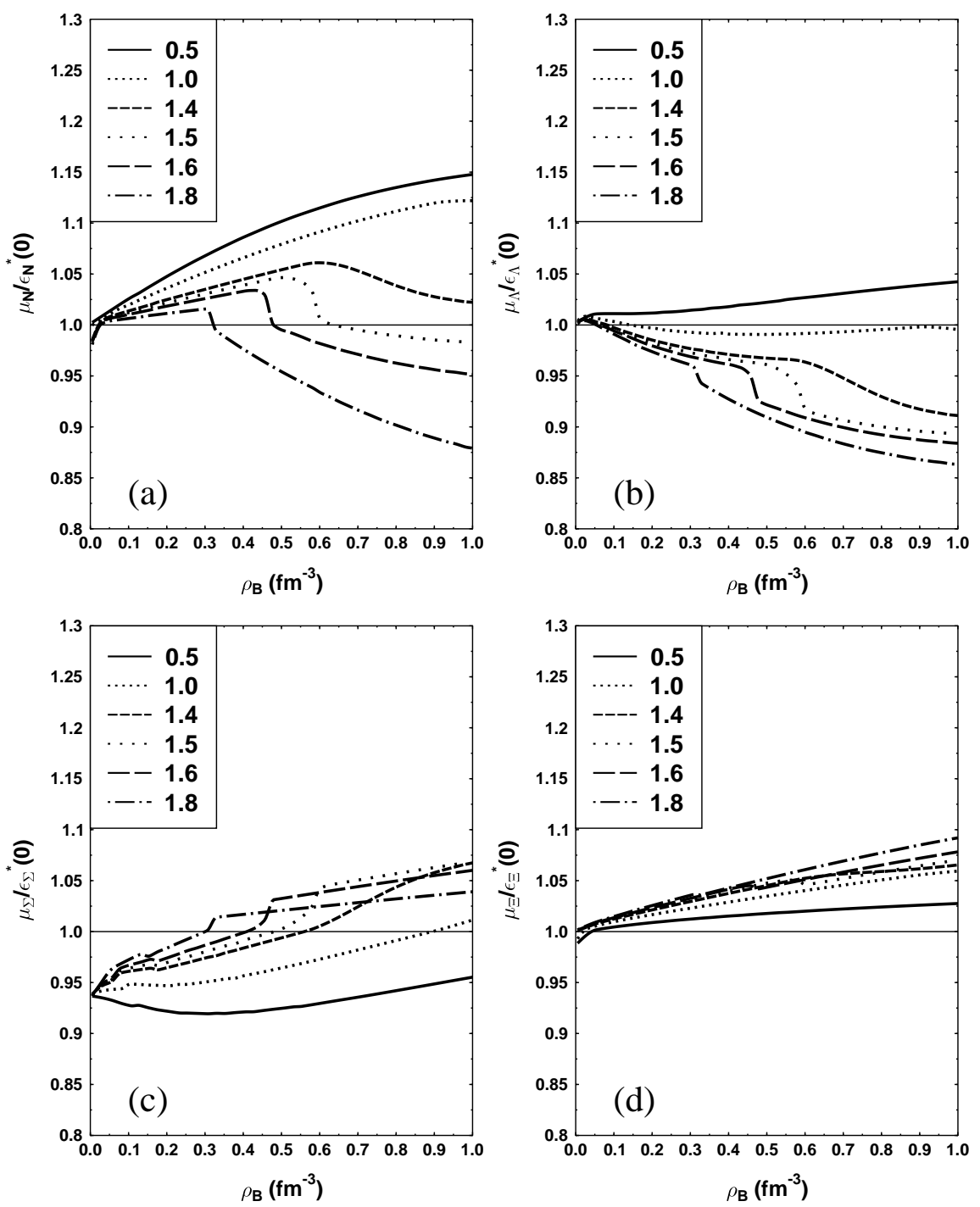

FIG. 11. The threshold production ratio $\mu_{i} / \epsilon_{i}^{*}\left(k_{F}=0\right)$ for each baryon species and for different strangeness fractions in MQMC-II. (a) $N$, (b) $\Lambda$, (c) $\Sigma$ and (d) $\Xi$. 

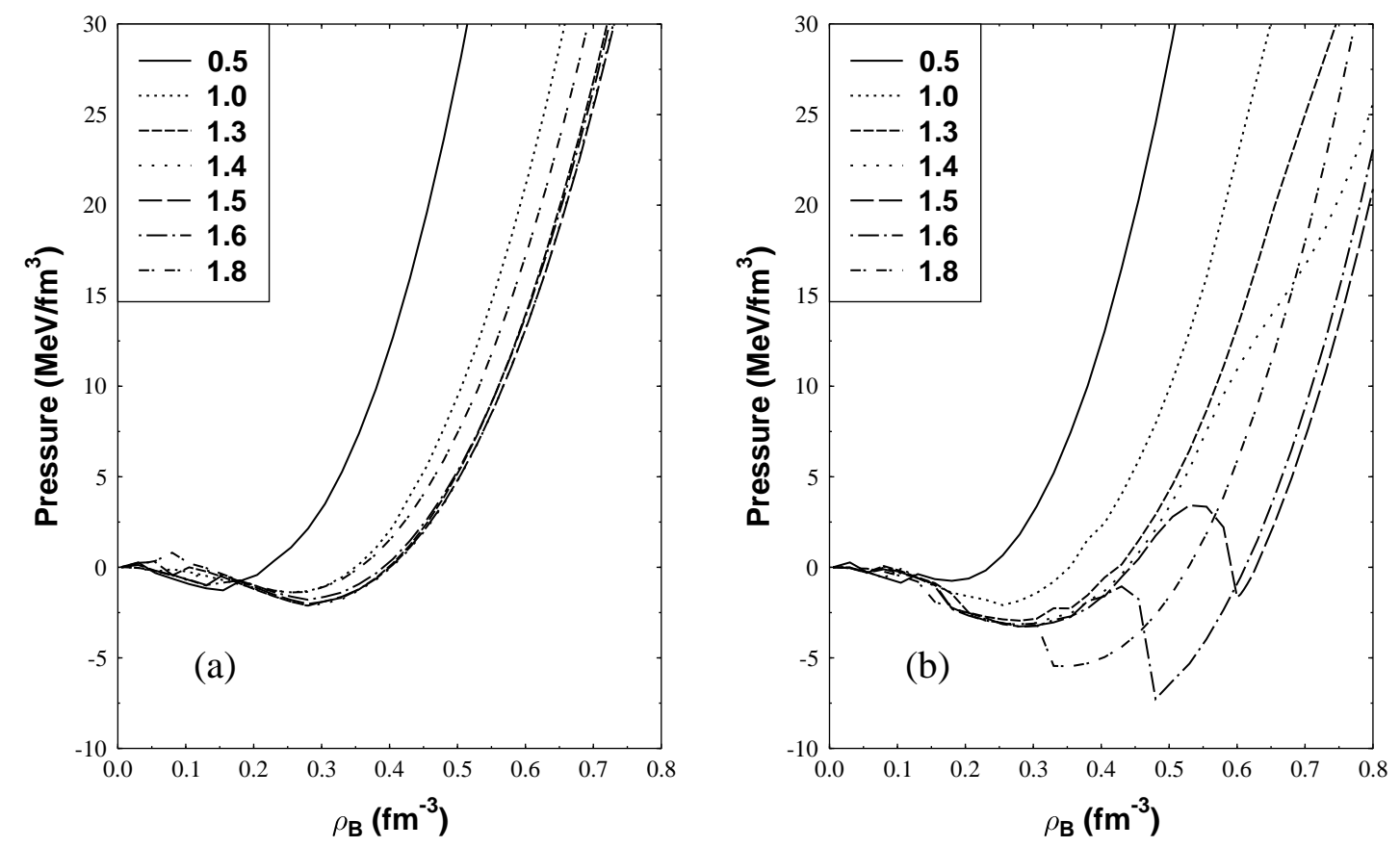

FIG. 12. Pressure of strange hadronic matter vs baryon density for different strangeness fractions in (a) MQMC-I and (b) MQMC-II. 